\title{
FACTORS ASSOCIATED WITH INDUCTION OF LABOUR AND PREGNANCY OUTCOMES IN 14 HEALTHCARE FACILITIES IN SRI LANKA
}

\author{
J. R. N. A. GUNAWARDANA ${ }^{1 *}$ AND S. S. N. PERERA ${ }^{2}$ \\ ${ }^{1}$ Department of Statistics \& Computer Science, Faculty of Science, \\ University of Kelaniya, Kelaniya, Sri Lanka \\ ${ }^{2}$ Department of Mathematics, Faculty of Science, University of Colombo, \\ Colombo 3, Sri Lanka
}

Received $21^{\text {st }}$ August $2018 /$ Accepted $13^{\text {th }}$ February 2019

\begin{abstract}
Induction of Labour (IOL) is an important practice that is carried out commonly in modern day obstetrics. In medium to large healthcare facilities in Sri Lanka, it is estimated that approximately $35.5 \%$ of all deliveries involve IOL. This research attempts to identify the factors that affect IOL and to assess the association between IOL and the pregnancy outcome. In this study, we considered 18309 women who were admitted to 14 healthcare facilities for delivery in 3 randomly selected provinces in Sri Lanka (Western, Southern and Eastern provinces), during July to October 2011. Multinomial Logistic Regression model (MLR) and Fuzzy Expert System (FES) were used to identify the factors that lead to IOL. The MLR model predicts IOL with a classification rate of $65.5 \%$ and the FES predicts IOL with an accuracy of $55.10 \%$. Maternal age, number of previous caesarian sections, number of previous births, estimated gestational age, Pre-Eclampsia, number of previous pregnancies, Placenta Preavia, Abruption Placenta, total number of neonates delivered, birth weight and Maternal Severity Index (MSI) were identified as Factors associated with IOL.
\end{abstract}

Corresponding author Email: nishaniamalka@gmail.com;

(D) https://orcid.org/0000-0002-5641-9332

DOI: http:://doi.org/0.4038/josuk.v12i0.8017

(c) (i) This is an open access article distributed under the terms of the Creative Commons Attribution 4.0 International License, which permits unrestricted use, distribution and reproduction in any medium provided the original work is properly credited. 
Neonatal status after seven days of life can also be predicted using the developed FES. FES is predictive of IOL and birth outcome, where if the FES score is between 0.8570 and 0.8854 , the patient will belong to the induced group and the baby would be alive after seven days of birth. This study concludes that, MLR and FES models can be used to predict IOL outcomes. These findings can be informative to healthcare providers when counselling women for labour induction and develop evidence-based protocols on IOL.

Keywords: Induction of labour, Multinomial logistic regression, Fuzzy expert system

\section{INTRODUCTION}

Induction of labour (IOL) is a common procedure in obstetrics (Laws, Li, \& Sullivan, 2010), (“National Institute for Health and Clinical Excellence: Guidance," 2008), and is defined as the initiation of labour by artificial means prior to its spontaneous onset at a viable gestational age, with the aim of achieving vaginal delivery in a pregnant woman with intact membranes. The procedure is not always successful and sometimes fails to achieve a safe vaginal delivery. It is estimated that in medium to large healthcare facilities in Sri Lanka, approximately 35.5\% of all deliveries involve IOL (World Health Organization, 2011).

The maternal mortality ratio in Sri Lanka declined to 30 deaths per 100,000 live births in 2015 from 75 deaths per 100,000 live births in 1990; this is an average annual reduction of 3.6\% over the 25-year period (WHO UNICEF UNFPA World Bank Group and United and United Nations Population Division, 2015). IOL is thought to be a factor in reducing maternal mortality by preventing maternal complications and improving pregnancy outcome (Hiluf \& Assefa, 2015). Little is known about factors that lead to IOL in Sri Lanka. This study therefore seeks to identify the factors leading to IOL, and assesses the association between IOL and pregnancy outcomes. The findings of this study can inform healthcare providers when counselling women for labour induction; develop evidence-based protocols on IOL within the local-context, and improve future quality of care provided for woman who need labour induction in hospitals. 
In the study by Hiluf and Assefa (Hiluf \& Assefa, 2015), a logistic regression analysis was used to assess the factors associated with IOL success. The study revealed that the age of women, Bishop score ${ }^{2}$, Apgar score ${ }^{3}$ and fetal heart beat at the beginning of induction were significantly associated with positive outcomes of IOL. Using multivariate linear regression, Gawade and Whitcomb (Gawade \& Whitcomb, 2012) concluded that prolonged second stage of labour, excessive gestational weight gain and cesarean delivery has been associated with adverse maternal and fetal outcomes. A cohort study in Canada (Bayrampour, Salmon, Vinturache, \& Tough, 2015), examined the associations between depressive and anxiety symptoms in the second and third trimesters and mode of delivery, epidural use and labour induction. Data were evaluated with multivariate multinomial and logistic regression analyses using a hierarchical modeling. No associations were found between antenatal depressive and anxiety symptoms and other obstetric interventions. Reis, Ortega, and Silveira (Reis, Ortega, \& Silveira, 2004), proposed a Fuzzy Expert System (FES) to predict the need for advanced neonatal resuscitation efforts in the delivery room. It was a structured fuzzy composition which provides a degree of association with the risk of occurrence of perinatal asphyxia ${ }^{4}$. The resulting relational matrix described the association between clinical factors and risk of perinatal asphyxia. Analyzing the inputs of the presence or absence of all clinical factors, the system returns the rate of risk of perinatal asphyxia as output. Nascimento and Ortega (Nascimento \& Ortega, 2002) introduced a fuzzy linguistic model for evaluating the risk of neonatal death. It was based on the fuzziness of two variables newborn birth weight and gestational age at delivery. The results were compared with expert opinion and the Fuzzy model captured the expert knowledge with a strong correlation.

This study is focused on developing a Multinomial Logistic Regression (MLR) model to assess the association between induction of labour and relative factors of IOL; and building a FES to predict the likelihood of undergoing labour induction.

\footnotetext{
${ }^{2}$ A Bishop score refers to a group of measurements used to determine whether a woman may have a successful vaginal delivery and whether labor ought to be induced.

${ }^{3}$ Apgar score is an objective score of the condition of a baby after birth. An Apgar score of 10 means an infant is in the best possible condition. A child with a score of 0 to 3 needs immediate resuscitation.

${ }^{4}$ Perinatal asphyxia happens when a baby's brain and other organs do not get enough oxygen during birth process.
} 


\section{METHODOLOGY}

\section{Data Collection}

In this study, we consider data of all women who were admitted to selected healthcare facilities for delivery, between July to October 2011 in 3 provinces. Data was attained from WHO (World Health Organization) Multi-Country Survey on Maternal \& New-born Health (Sri Lanka) database. Healthcare facilities were identified through a multistage sampling method. The first sampling stage randomly selected 3 provinces in a probability proportional to the population size manner: namely Western, Eastern and Southern provinces. Once provinces were selected, healthcare facilities with a minimum of 1000 deliveries per year were randomly selected from each of the provinces. Nearly 18300 deliveries, in 14 facilities, were studied during a 2-3-month period.

\section{Descriptive Analysis}

Onset of labour is likely to be present in three ways as follows:

1) Spontaneous onset of labour - natural labour or no induction of labour, 2)

Labour induction - labour initiated following the use of formal of induction of labour including pharmacological and mechanical methods, and

3) No labour - delivery by caesarean section before labour.

All pregnancy related characteristics were compared between all three onsets of labour categories, using cross-tabulations, bar charts and histograms.

\section{Preliminary Analysis}

Chi-square test for independence, Fisher's exact test and LOWESS / LOESS curve were used during preliminary analysis to identify the relationship between IOL and predictor variables. Based on the literature and preliminary analysis, predictor variables were identified to build the MLR and FES models.

Identified predictor variables are as follows (Table 1). 
Table 1: Identified predictor variables of IOL

\begin{tabular}{|l|l|}
\hline \multicolumn{1}{|c|}{ Numerical } & \multicolumn{1}{c|}{ Categorical } \\
\hline Maternal age in years & Fatal presentation at delivery \\
\hline $\begin{array}{l}\text { Number of pregnancies (including } \\
\text { current pregnancy) }\end{array}$ & Placenta Praevia \\
\hline $\begin{array}{l}\text { Number of previous births (excluding current } \\
\text { delivery) }\end{array}$ & Abruption Placenta ${ }^{5}$ \\
\hline Number of previous caesarean sections & Pre-Eclampsia $^{6}$ \\
\hline $\begin{array}{l}\text { Best estimate of gestational age at delivery or } \\
\text { abortion }\end{array}$ & Anaemia \\
\hline Total number of neonates delivered & Thrombocytopenia $^{7}$ \\
\hline Birth weight & \\
\hline
\end{tabular}

\section{Multinomial Logistic Regression (MLR)}

Multinomial logistic regression (MLR) is used to predict a nominal dependent variable given one or more independent variables. It is sometimes considered an extension of binomial logistic regression to allow for a dependent variable with more than two categories. As with other types of regression, MLR can have nominal and/or continuous independent variables and interactions between independent variables to predict the dependent variable ("How to perform a Multinomial Logistic Regression in SPSS Statistics (Laerd Statistics," n.d.).

Suppose $Y$ is a categorical (polychromous) response variable with $C$ categories, taking on values $1,2, \ldots, C-1$ and there are $k$ explanatory variables $X_{1}, X_{2}, \ldots, X_{k}$.

Then the multinomial logistic regression model for each non-reference category $j=1,2, \ldots$ , $C-1$ against the reference category 0 , is given by:

$$
\log \left(\frac{\pi^{(j)}}{(0)}\right)=\beta_{0} \pi^{(j)}+\beta_{1}^{(j)} X_{1}+\cdots+{ }_{\beta k} X_{k},
$$

where $\beta_{0}(j), \beta_{1}(j), \ldots, \beta_{k}^{(j)}$ are unknown population parameters and $\pi^{(j)}$ is the probability of getting $j^{\text {th }}$ category, and is given by:

$$
\pi^{(j)}=\frac{\exp \left(\sum_{i=0}^{k} \beta_{i}^{(j)} X_{i}\right)}{1+\exp \left(\sum_{i=0}^{k} \quad \beta_{i} X_{i}\right)}
$$

\footnotetext{
${ }^{5}$ Placenta Praevia occurs when a baby's placenta partially or totally covers the opening in the mother's cervix.

${ }^{5}$ Abruption Placenta occurs when the placenta separates from the wall of the uterus prior to the birth of the baby.

${ }^{6}$ Pre-Eclampsia is a serious condition with high blood pressure and problems with one or more organs.

${ }^{7}$ Thrombocytopenia is defined as a platelet count of less than 50000 per ml.
} 


\section{Checking the Assumptions for MLR}

Assumptions of multicollinearity, linearity and outliers for MLR models were checked using standard error, scatterplot of difference between observed and predicted probabilities versus predicted probability and studentized residual respectively in this study.

\section{Goodness of Fit Test Statistics for MLR}

Deviance, Chi-square goodness of fit test, likelihood ratio test, McFadden's R², Cox and Snell pseudo $\mathrm{R}^{2}$, Nagelkerke pseudo $\mathrm{R}^{2}$ and proportional by chance accuracy criteria were used to assess the goodness of fit of the fitted MLR models.

\section{Fuzzy Expert System (FES)}

A Fuzzy Expert System (FES) is an expert system that uses fuzzy logic through a collection of fuzzy membership functions and rules, instead of Boolean logic to reason about data (Azim, Jaffar, \& Mirza, 2014).

\section{Fuzzy Sets and Membership Functions}

Fuzzy logic is a logic based on fuzzy sets, that is sets of elements or objects characterized by truth-values in the $[0,1]$ interval. The function that assigns a number in $[0$, 1] to each element of the universe of discourse of a fuzzy set is called the membership function (Pappis, 2005).

Let $X$ denote the universe of discourse of a fuzzy set. $A$ is completely characterized by its membership function $\mu_{A}$ :

$$
\mu_{A}: X \rightarrow[0,1]
$$

and is defined as a set of pairs:

$$
A=\left\{\left(x, \mu_{A}(x)\right)\right\}
$$

Developed membership functions for each IOL predictor variable are given below in Table 2. 
Table 2: Membership function of each input IOL predictor variable

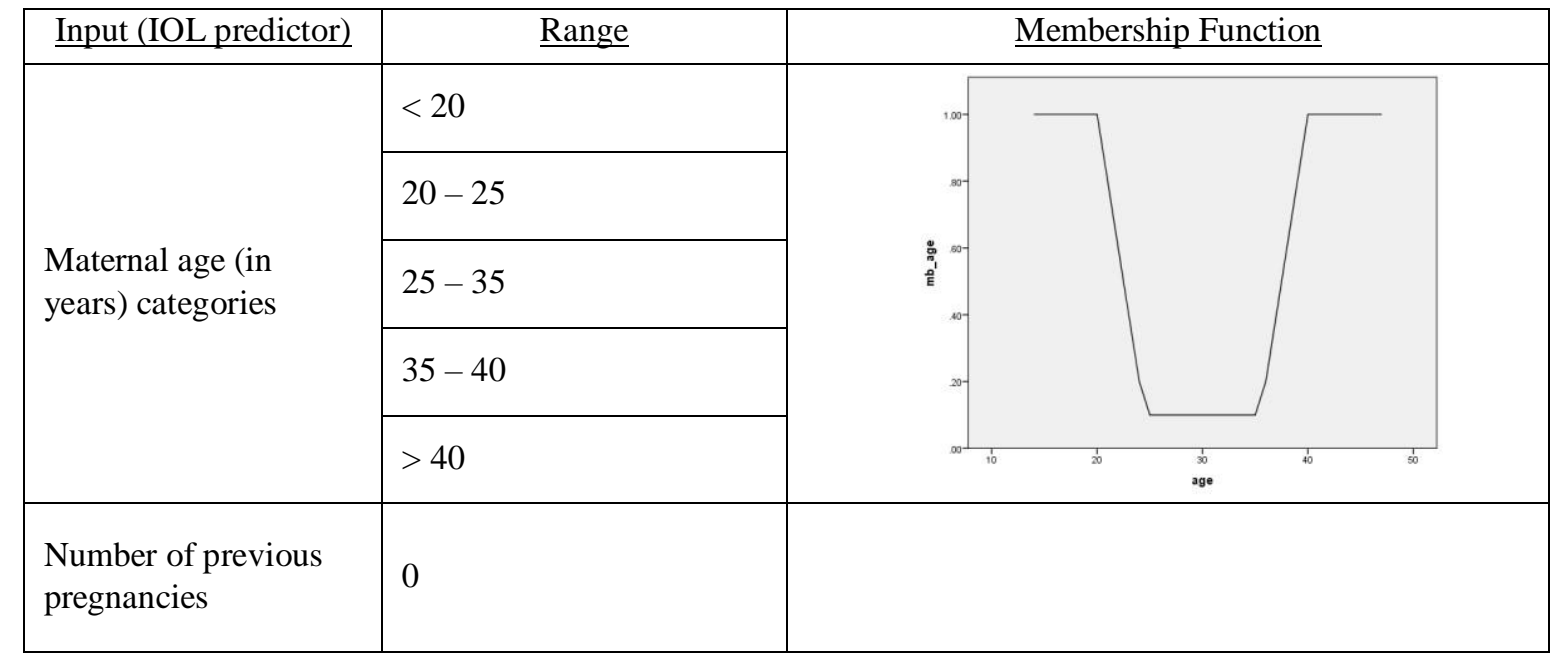

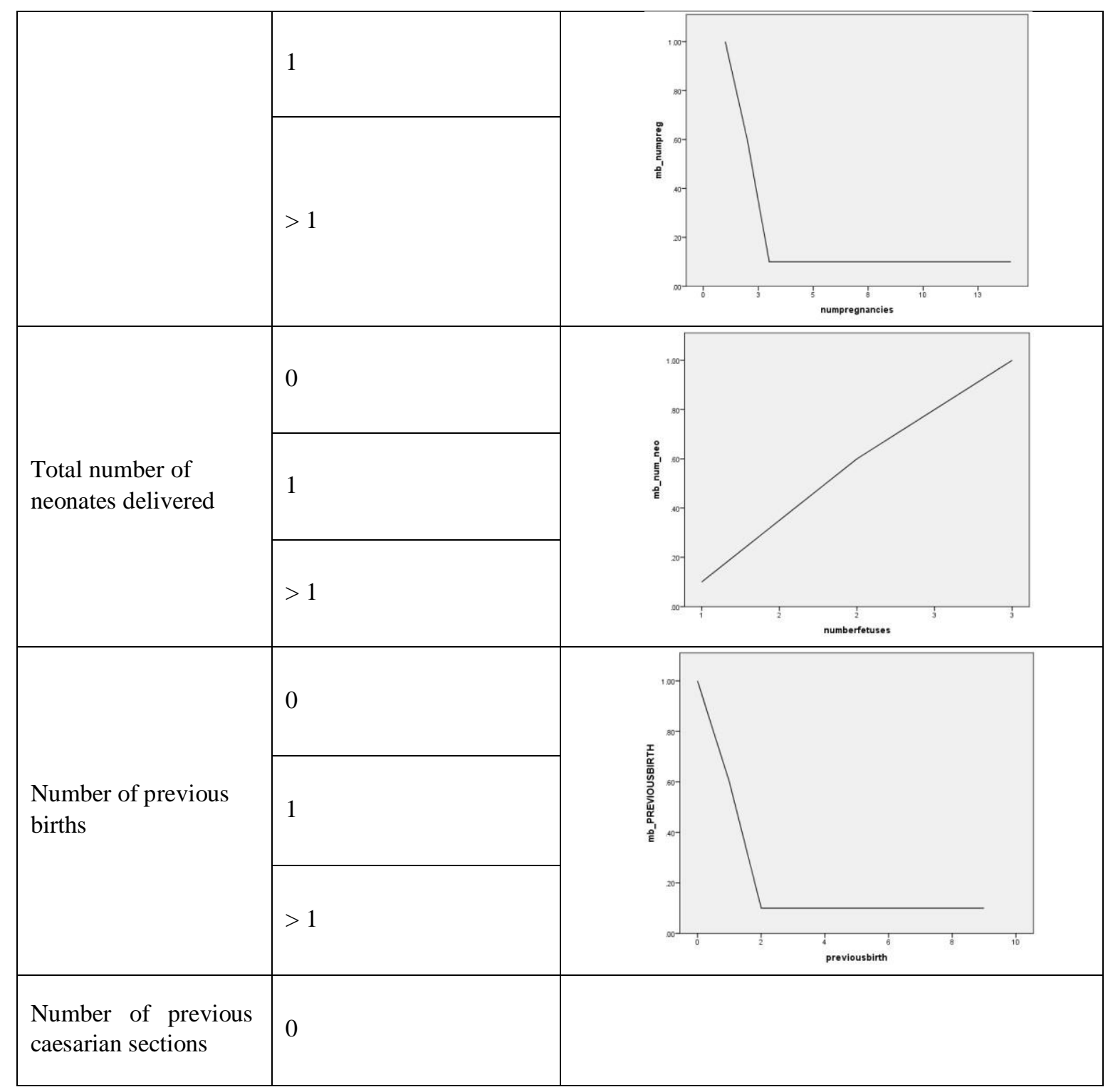




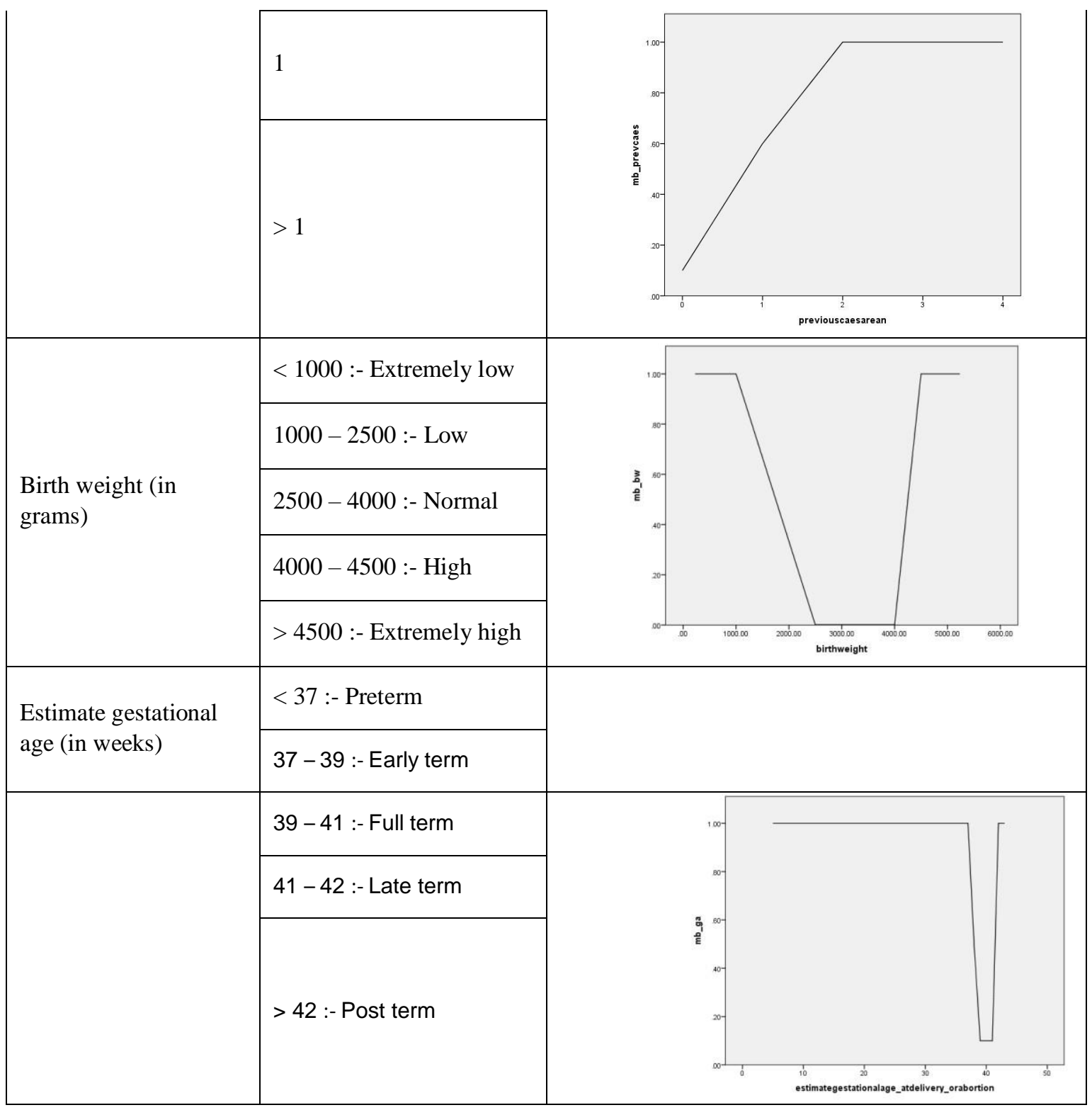

\section{Fuzzy Rules}

Let there be a multiple-input-single-output rule base with $N$ rules. The $i^{\text {th }}$ rule is given by

$$
\text { If } A_{i 1} \text { and } A_{i 2} \text { and } \ldots \text { and } A_{\text {in }} \text { then } B_{i}
$$

where $n$ is the number of input variables $x_{i}, A_{i j}$ is the fuzzy set of input variable $x_{j}$ in the $i^{\text {th }}$ rule, and $B_{i}$ is the fuzzy set of output variable $y_{j}$ in the $i^{\text {th }}$ rule (Pappis, 2005).

Described below are fuzzy rules which were used for the model building procedure.

1) If one input factor is severe, and the other input factor is also severe, then the outcome should be more severe than both factors.

2) If one input factor is moderate, and the other input factor is severe, then the outcome should be either between moderate and severe, or can be more severe than the severe input factor. 


\section{Checking Goodness of Fit Test Statistics for FES}

Receiver Operating Characteristic (ROC) Curves and Area Under the Curve (AUC) were used to assess the performance of the fitted model. Cut-off values were determined using the graph of distance (D) versus test score.

Sensitivity, specificity, accuracy, Misclassification Rate (MR), Positive Predictive Value (PPV), Negative Predictive Value (NPV), Positive Likelihood Ratio (LR+), Negative Likelihood Ratio (LR-) and Relative Risk (RR) were indices used for evaluation of the developed score.

\section{RESULTS}

\section{Descriptive Analysis}

Table 3: Cross tabulations between onset of labour and categorical characteristic variables

\begin{tabular}{|c|c|c|c|c|}
\hline \multirow{2}{*}{$\frac{\text { Characteristic }}{\underline{\text { variable }}}$} & \multirow[b]{2}{*}{ Category } & \multicolumn{3}{|c|}{ Characteristic variable } \\
\hline & & $\begin{array}{c}\text { Spontaneous } \\
\text { labour }\end{array}$ & Induced labour & No labour \\
\hline \multirow{2}{*}{$\begin{array}{l}\text { Maternal } \\
\text { complications }\end{array}$} & Yes & 283 & 272 & 289 \\
\hline & No & 7703 & 6073 & 3489 \\
\hline \multirow{2}{*}{$\begin{array}{l}\text { Maternal vital } \\
\text { status at discharge } \\
\text { from hospital }\end{array}$} & Alive & 7986 & 6344 & 3777 \\
\hline & Dead & 0 & 1 & 1 \\
\hline \multirow{3}{*}{$\begin{array}{l}\text { Final mode of } \\
\text { delivery/abortion }\end{array}$} & Vaginal delivery & 6774 & 5172 & 0 \\
\hline & Caesarean section & 1211 & 1169 & 3778 \\
\hline & Other & 1 & 4 & 0 \\
\hline \multirow{3}{*}{$\begin{array}{l}\text { Fatal presentation } \\
\text { at delivery }\end{array}$} & Cephalic & 7802 & 6305 & 3318 \\
\hline & Breech & 187 & 49 & 332 \\
\hline & Other & 42 & 20 & 84 \\
\hline \multirow{3}{*}{$\begin{array}{l}\text { Vital status of the } \\
\text { neonate at birth }\end{array}$} & Alive & 8014 & 6314 & 3831 \\
\hline & Fresh stillbirth & 18 & 22 & 9 \\
\hline & $\begin{array}{l}\text { Macerated } \\
\text { stillbirth }\end{array}$ & 22 & 9 & 11 \\
\hline \multirow{2}{*}{$\begin{array}{l}\text { New born status at } \\
\text { hospital discharge } \\
\text { or on the seventh } \\
\text { day of life }\end{array}$} & Alive & 7954 & 6296 & 3796 \\
\hline & Dead & 47 & 15 & 30 \\
\hline
\end{tabular}

Some important descriptive results are given in Table 3 and Table 4. Table 3 summarizes about categorical variables while, Table 4 illustrates histograms of numerical variables. 
Table 4: Histograms between onset of labour and numerical characteristic variables

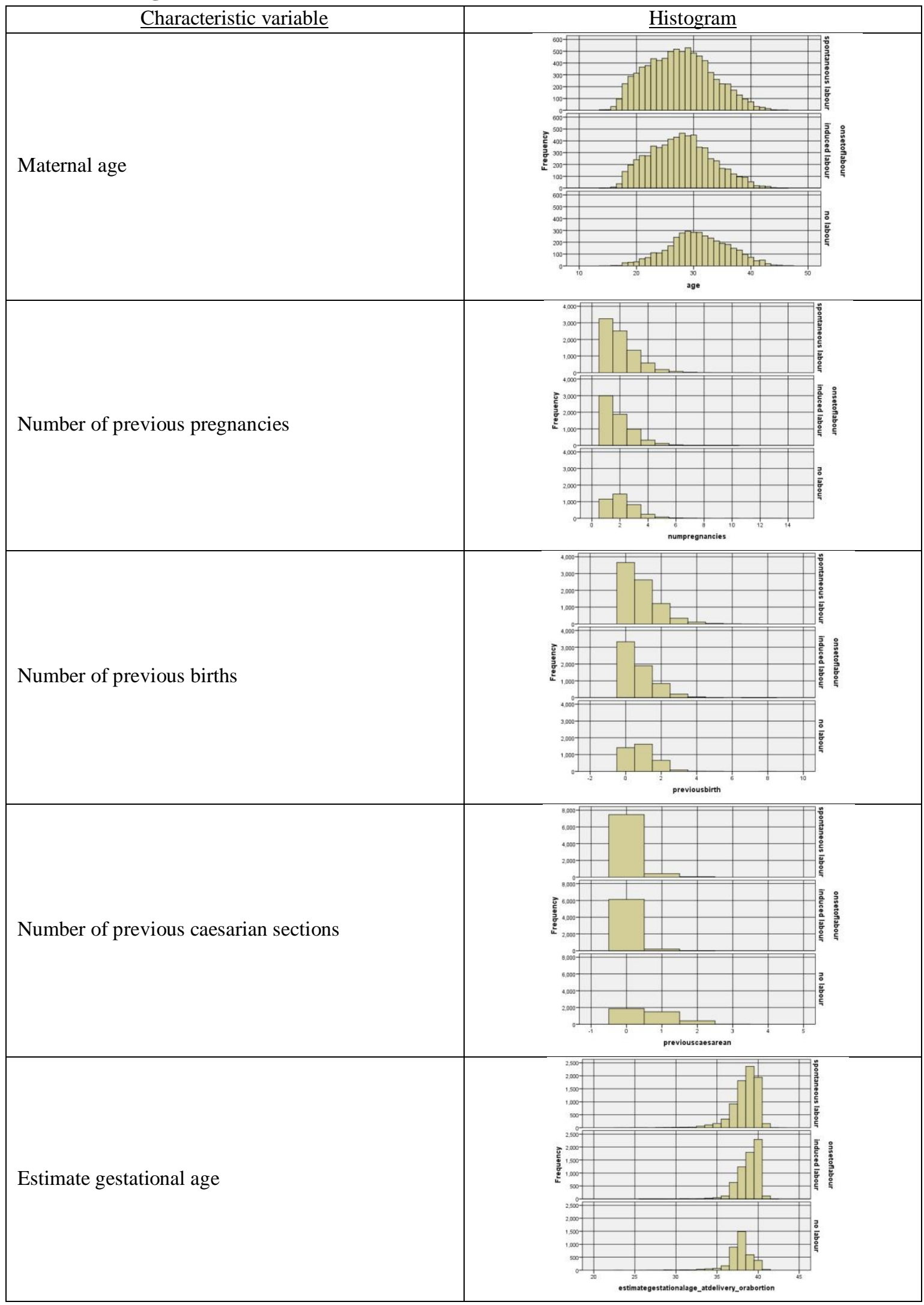




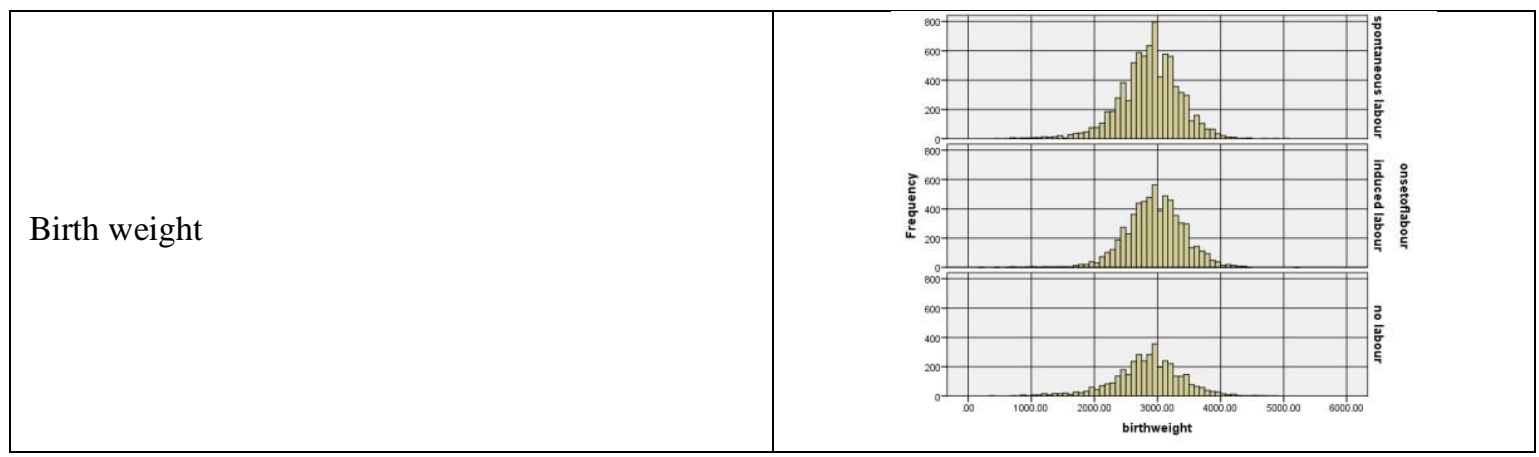

\section{Multinomial Logistic Regression Model}

Multinomial Logistic Regression models were assessed for spontaneous labour and induced labour. The fitted models are given below:

(1) MLR model for spontaneous labour:

$\log [P($ onsetoflabour $=$ spontaneous $)]$

$$
\begin{aligned}
& P(\text { onsetoflabour }=\text { nolabour }) \\
& \quad=-1.493+0.061 \text { age })(\text { prevcaes })-0.090(\text { prevcaes })(g a) \\
& \quad-0.726[\text { preeclampsia }=1](\text { numpreg }) \\
& \quad-2.103[\text { preeclampsia }=2](\text { numpreg }) \\
& \quad+0.787[\text { placentapraevia }=1](\text { numpreg }) \\
& \quad+0.818[\text { preeclampsia }=1](\text { previousbirth }) \\
& \quad+2.313[\text { preeclampsia }=2](\text { previousbirth }) \\
& \quad+0.034[\text { abruptionplacenta }=1](\text { ga })-0.003(\text { age })(g a)
\end{aligned}
$$

1) MLR model for induced labour: $\log$

$$
P(\text { onsetoflabour }=\text { induced })
$$

$$
\begin{aligned}
& P(\text { onsetoflabour }=\text { nolabour }) \\
& \quad=-10.705+0.172(\text { age })(\text { prevcaes })-0.220(\text { prevcaes })(\mathrm{ga}) \\
& \quad-1.350[\text { preeclampsia }=1](\text { numpreg }) \\
& \quad-1.981[\text { preeclampsia }=2](\text { numpreg }) \\
& +1.143[\text { placentapraevia }=1](\text { numpreg }) \\
& \quad+0.859[\text { preeclampsia }=1](\text { previousbirth }) \\
& +1.570[\text { preeclampsia }=2](\text { previousbirth }) \\
& \quad+0.038[\text { abruptionplacenta }=1](\text { ga })-0.002(\text { age })(\mathrm{ga})
\end{aligned}
$$

where:

ga, age, prevcaes, numpreg, previousbirth, preeclampsia, placentapraevia and abruptionplacenta are estimate gestational age, maternal age, number of previous caesarian sections, number of previous pregnancies, number of previous births, PreEclampsia, Placenta Praevia and Abruption Placenta respectively. 


\section{Checking for MLR Assumptions}

All standard error values related to predictors are less than five, indicating that there are no multicollinearity problems.
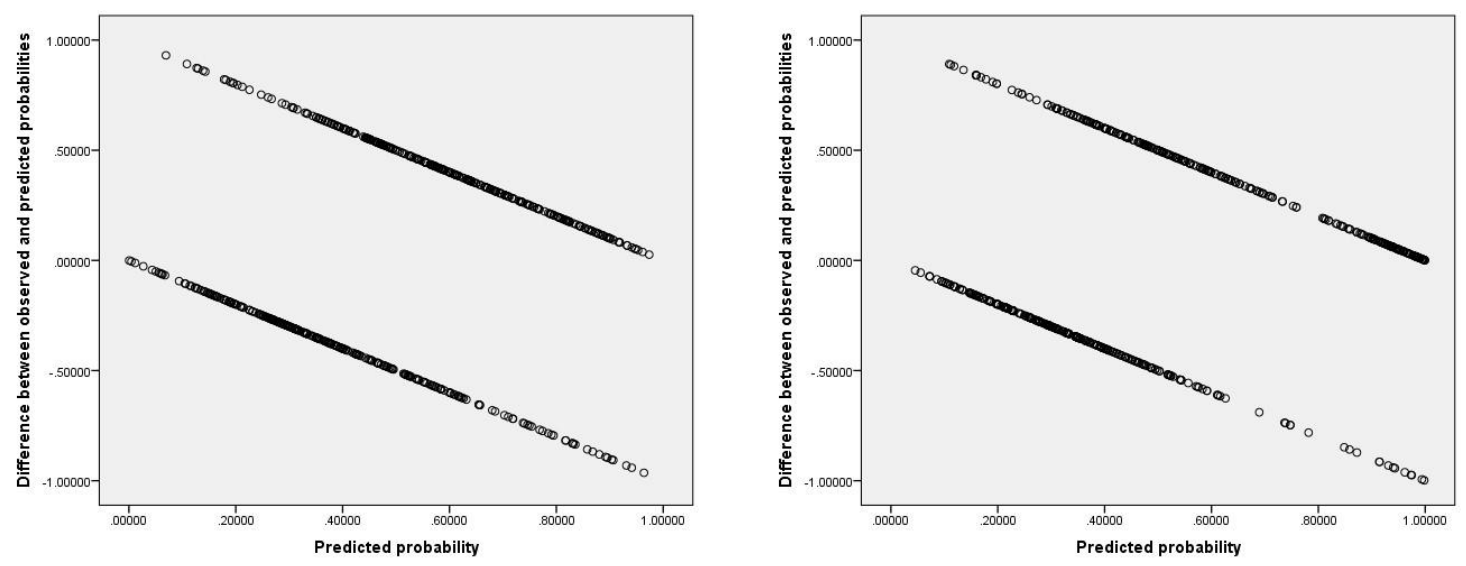

Figure 1: Scatter plot of residuals versus Figure 2: Scatter plot of residuals versus predicted probabilities for spontaneous predicted probabilities for induced labour and no labour categories labour and no labour categories

Figure 1 illustrates scatter plot of residuals versus predicted probabilities for spontaneous labour and no labour categories and Figure 2 displays the same for induced labour and no labour categories. Since the points along the scatterplots of Figure 1 and Figure 2, are symmetric both above and below a straight line, with observations being equally spaced out along the line, the assumption of linearity is assumed. Outliers indicate the presence of severe maternal cases. Therefore, outliers are not removed throughout the model building procedure.

\section{Goodness of Fit for MLR Model}

Table 5: Model fitting information for MLR model

\begin{tabular}{|l|l|l|l|l|}
\hline \multicolumn{2}{|l|}{ Model Fitting Information } \\
\hline Model & $\begin{array}{l}\text { Model Fitting } \\
\text { Criteria }\end{array}$ & \multicolumn{2}{l|}{ Likelihood Ratio Tests } \\
\cline { 2 - 5 } & $\begin{array}{l}-2 \\
\text { Likelihood Log }\end{array}$ & Chi-Square & df & Sig. \\
\hline Intercept Only & 2052.874 & & & \\
\hline Final & 1670.471 & 382.403 & 22 & .000 \\
\hline
\end{tabular}

Model fitting information for the MLR model is displayed in Table 5. Given that the p-value is less than 0.05 , the full model statistically and significantly predicts onset of labour better than the intercept-only model alone. 
Table 6: Goodness of fit for MLR model

\begin{tabular}{|l|l|l|l|}
\hline \multicolumn{4}{|l|}{ Goodness-of-Fit } \\
\hline & Chi-Square & df & Sig. \\
\hline Pearson & 1969.551 & 1842 & .119 \\
\hline Deviance & 1670.471 & 1842 & .998 \\
\hline
\end{tabular}

Table 6 summarizes the goodness of fit for the MLR model. The p-value is greater than 0.05 , therefore we do not reject the null hypothesis of Chi-Square goodness of fit test, and conclude that data are consistent with the specified distribution.

Table 7: $\mathbf{R}^{2}$ values for MLR model

\begin{tabular}{|l|c|}
\hline \multicolumn{2}{|c|}{ Pseudo R-Square } \\
\hline Cox and Snell & .336 \\
\hline Nagelkerke & .378 \\
\hline McFadden & .186 \\
\hline
\end{tabular}

$\mathrm{R}^{2}$ values for the model are listed in Table 7. Nagelkerke $\mathrm{R}^{2}$ is 0.378 , which implies that $37.8 \%$ variability of onset of labour is explained by the explanatory variables.

Of much greater importance are the results presented in the likelihood ratio tests shown in Table 8. All p-values of likelihood ratio tests are less than 0.05 , indicating that all variables in the final model are statistically significant.

Table 8: Likelihood ratio tests for MLR model

\begin{tabular}{|c|c|c|c|c|}
\hline \multicolumn{5}{|c|}{ Likelihood Ratio Tests } \\
\hline \multirow[t]{2}{*}{ Effect } & \multirow{2}{*}{\begin{tabular}{|ll}
$\begin{array}{c}\text { Model Fitting } \\
\text { Criteria }\end{array}$ \\
-2 Log Likelihood \\
of Reduced Model
\end{tabular}} & \multicolumn{3}{|c|}{ Likelihood Ratio Tests } \\
\hline & & $\begin{array}{l}\text { Chi- } \\
\text { Square }\end{array}$ & df & Sig. \\
\hline Intercept & 1718.285 & 47.815 & 2 & .000 \\
\hline age $*$ prevcaes & 1683.075 & 12.604 & 2 & .002 \\
\hline prevcaes $*$ ga & 1697.287 & 26.816 & 2 & .000 \\
\hline preeclampsia * numpreg & 1703.738 & 33.267 & 2 & .000 \\
\hline placentapraevia * numpreg & 1727.370 & 56.899 & 2 & .000 \\
\hline preeclampsia $*$ previousbirth & 1710.007 & 39.536 & 4 & .000 \\
\hline abruptionplacenta $*$ ga & 1679.909 & 9.438 & 2 & .009 \\
\hline age $*$ ga & 1709.238 & 38.767 & 2 & .000 \\
\hline
\end{tabular}


Table 9: Classification table for MLR model

\begin{tabular}{|c|c|c|c|c|}
\hline \multicolumn{5}{|c|}{ Classification } \\
\hline \multirow[t]{2}{*}{ Observed } & \multicolumn{4}{|l|}{ Predicted } \\
\hline & $\begin{array}{c}\text { spontaneous } \\
\text { labour }\end{array}$ & $\begin{array}{l}\text { induced } \\
\text { labour }\end{array}$ & no labour & $\begin{array}{l}\text { Percent } \\
\text { Correct }\end{array}$ \\
\hline $\begin{array}{l}\text { spontaneous } \\
\text { labour }\end{array}$ & 119 & 136 & 64 & $37.3 \%$ \\
\hline induced labour & 71 & 211 & 40 & $65.5 \%$ \\
\hline no labour & 44 & 49 & 201 & $68.4 \%$ \\
\hline $\begin{array}{l}\text { Overall } \\
\text { Percentage }\end{array}$ & $25.0 \%$ & $42.4 \%$ & $32.6 \%$ & $56.8 \%$ \\
\hline
\end{tabular}

Table 9 outline the classification table for the MLR model. From Table 9:

Proportional by Chance Accuracy Rate $=0.3411^{2}+0.3433^{2}+0.3144^{2}=0.333$

Proportional by Chance Accuracy Criteria $=1.25 \times 0.333=41.71 \%$

The overall percentage for IOL classification with the MLR model is $56.8 \%$, which is greater than the proportional by chance accuracy criterion of $41.71 \%$. Hence the criterion for classification accuracy is satisfied.

\section{Fuzzy Expert System}

The fitted score of the fuzzy expert system is given by:

score $=\left(\left(\left(m b \_n u m n e o^{0.6} m b \_b w^{0.3}\right)^{0.3} m b \_M S I^{0.1}\right)^{0.2} m b \_g a^{0.2}\right)^{0.1} \times$

$\left(\left(m b \_a g e^{0.1} \text { mb_prevcaes }{ }^{0.5}\right)^{0.6}\left(m b \_n u m p r e g^{0.1} m_{\text {b_previousbirth }}{ }^{0.5}\right)^{0.1}\right)^{0.1}$

where:

mb_numneo, mb_bw, mb_MSI, mb_ga, mb_age, mb_prevcaes, mb_numpreg and previousbirth are the membership functions of total number of neonates delivered, birth weight, maternal severity index, estimate gestational age, maternal age, number of previous caesarian sections, number of previous pregnancies and number of previous births respectively. 


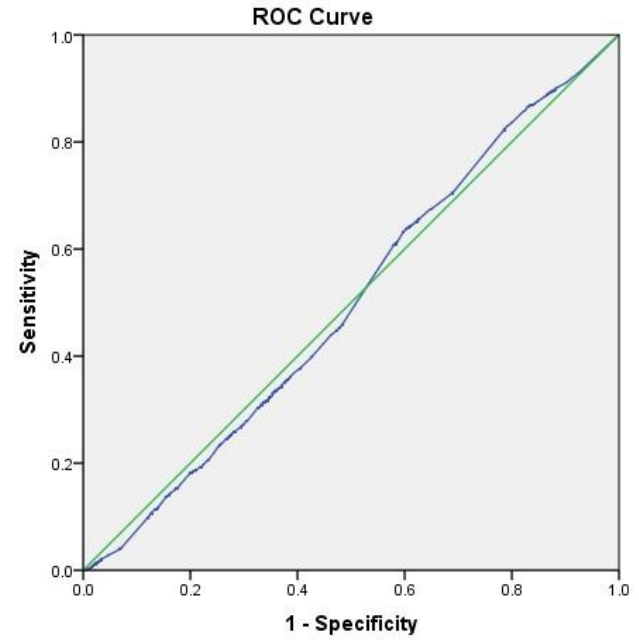

Figure 3: ROC curve of the score for Figure 4: ROC curve of the score for

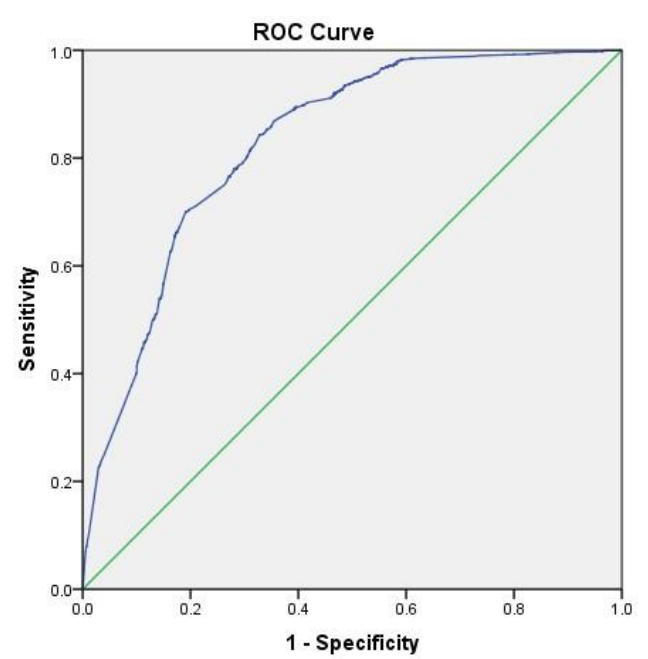
spontaneous labour and induced labour induced labour and no labour

The ROC curve of the score for spontaneous labour and induced labour is illustrated in Figure 3 and is spread along the AUC $=0.5$ line while the ROC curve of the score for induced labour and no labour is displayed in Figure 4 and is spread above AUC $=0.5$ line. AUC for spontaneous labour and induced labour is displayed in Table 10 sand is equal to 0.498, which is very close to 0.5. Table 11 illustrates AUC for spontaneous labour and induced labour, where AUC is 0.827 , which is statistically significant.

Table 10: AUC for spontaneous labour and induced labour

\begin{tabular}{|c|c|c|c|c|}
\hline \multicolumn{5}{|c|}{ Area Under the Curve } \\
\hline \multicolumn{5}{|c|}{ Test Result Variable(s): score } \\
\hline \multirow[t]{2}{*}{ Area } & \multirow[t]{2}{*}{$\begin{array}{l}\text { Std. } \\
\text { Error }^{\mathrm{a}}\end{array}$} & \multirow[t]{2}{*}{$\begin{array}{l}\text { Asymptotic } \\
\text { Sig. }{ }^{b}\end{array}$} & \multicolumn{2}{|c|}{$\begin{array}{c}\text { Asymptotic } 95 \% \text { Confidence } \\
\text { Interval }\end{array}$} \\
\hline & & & Lower Bound & Upper Bound \\
\hline .498 & .005 & .716 & .487 & .509 \\
\hline
\end{tabular}

Table 11: AUC for induced labour and no labour

\begin{tabular}{|c|c|c|c|c|}
\hline \multicolumn{5}{|c|}{ Area Under the Curve } \\
\hline \multicolumn{3}{|c|}{ Test Result Variable(s): score } \\
\hline Area & Std. Error & Asymptoti \\
& & c Sig. $^{\text {b }}$ & \multicolumn{2}{|c|}{$\begin{array}{c}\text { Asymptotic 95\% Confidence } \\
\text { Interval }\end{array}$} \\
\cline { 4 - 5 } & & & Lower Bound & Upper Bound \\
\hline .827 & .005 & .000 & .817 & .837 \\
\hline
\end{tabular}




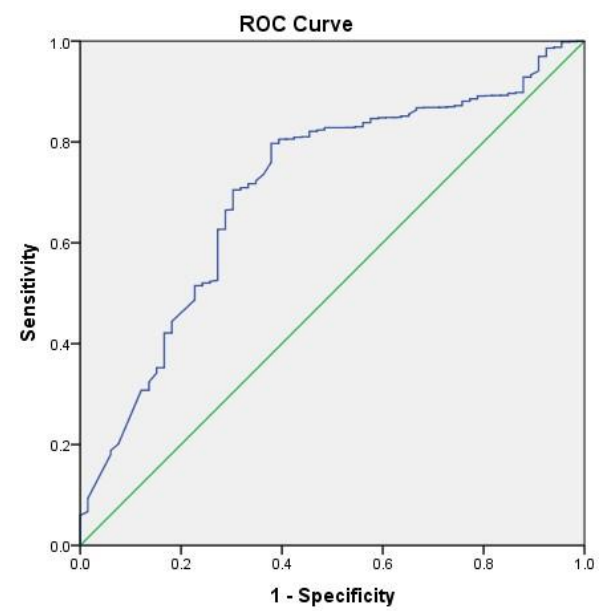

Figure 5: ROC curve of the score for neonatal status

Table 12: AUC for neonatal status

\begin{tabular}{|c|c|c|c|c|}
\hline \multicolumn{5}{|c|}{ Area Under the Curve } \\
\hline Test Result Variable(s): score \\
\hline Area & Std. Error & $\begin{array}{c}\text { Asymptoti } \\
\text { c Sig. }\end{array}$ & \multicolumn{2}{|c|}{$\begin{array}{c}\text { Asymptotic 95\% Confidence } \\
\text { Interval }\end{array}$} \\
\cline { 4 - 5 } & & & Lower Bound & Upper Bound \\
\hline .706 & .032 & .000 & .644 & .768 \\
\hline
\end{tabular}

The ROC curve of the score for neonatal status is displayed in

Figure 5 and is spread above the AUC $=0.5$ line. Table 12 describes AUC for neonatal status. AUC for neonatal status is 0.706 , which is statistically significant.
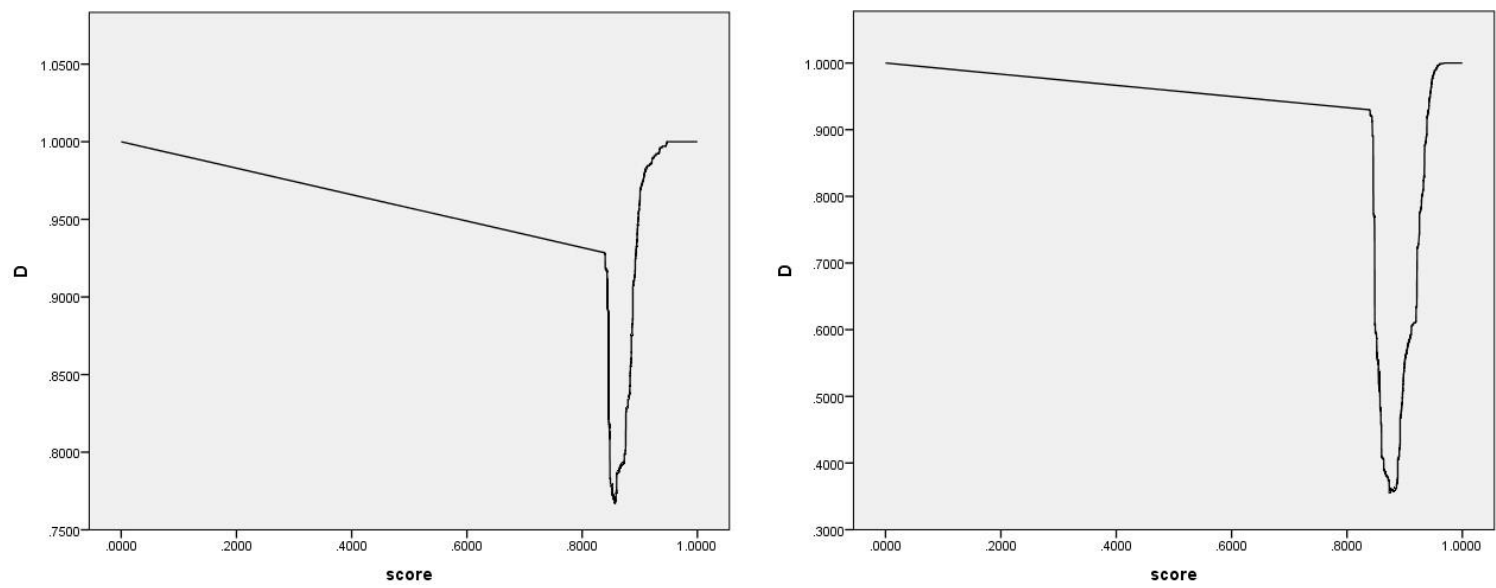

Figure 6: Graph of D versus test score

Figure 7: Graph of $D$ versus test score for spontaneous labour and induced for induced labour and no labour labour categories categories 
Figure 6 illustrates the graph of D versus test score for spontaneous labour and induced labour categories. The minimum distance for spontaneous labour and induced labour is obtained when score is equal to 0.8570 . Therefore, the cut-off for value for spontaneous labour and induced labour is 0.8570 . The graph of $\mathrm{D}$ versus test score for induced labour and no labour categories is displayed in Figure 7. The minimum distance for induced labour and no labour is obtained when score is equal to 0.8881 . Therefore, the cut-off for value for induced labour and no labour is 0.8881 .

Hence the criteria for onset of labour category is as follows: if score $<0.8570$ then onset of labour category is spontaneous; if $0.8570 \leq$ score $\leq 0.8881$ then onset of labour category is induced, and if score $>0.8881$ then onset of labour category is no labour.

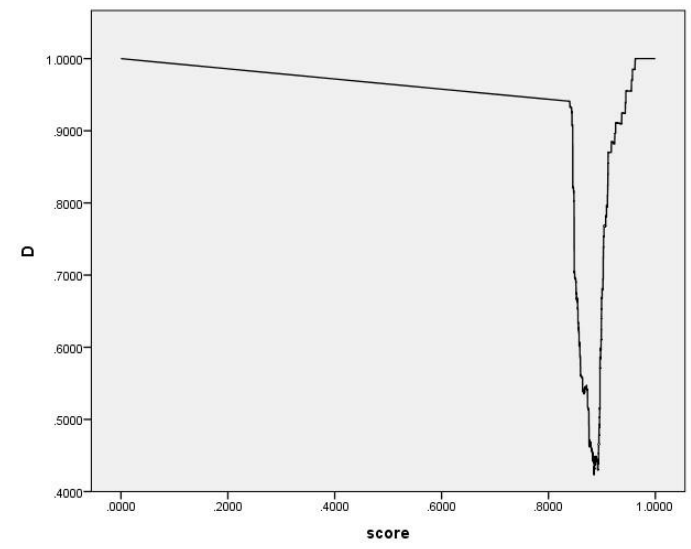

Figure 8: Graph of D versus test score for neonatal status

Figure 8 displays the graph of $\mathrm{D}$ versus test score for neonatal status. The minimum distance for neonatal status is obtained when score is equal to 0.8854 . Therefore, the cut-off for value for neonatal status is 0.8854 .

Hence the criteria for neonatal status is as follows: if score $\leq 0.8854$ then neonatal status is alive, and if score $>0.8854$ then neonatal status is dead.

\section{Evaluation Indices}

Table 13: Crosstabulation of observed and predicted frequencies of IOL

\begin{tabular}{|l|l|c|c|c|}
\hline \multicolumn{4}{|c|}{ predicted * observed Crosstabulation } \\
\hline \multicolumn{2}{|c|}{ Count } & \multicolumn{2}{|c|}{ observed } & \multirow{2}{*}{ Total } \\
\cline { 3 - 5 } & with IOL & $\begin{array}{c}\text { without } \\
\text { IOL }\end{array}$ & \\
\hline \multirow{2}{*}{ predicted } & $\begin{array}{l}\text { with } \\
\text { IOL }\end{array}$ & 474 & 824 & 1298 \\
\cline { 2 - 5 } & $\begin{array}{l}\text { without } \\
\text { IOL }\end{array}$ & 790 & 1507 & 2297 \\
\hline Total & & 1264 & 2331 & 3595 \\
\hline
\end{tabular}


Table 13 summarizes cross tabulation of observed and predicted frequencies of IOL. From Table 13, the calculated indices for IOL are as follows:

Sensitivity $=37.50 \%$, Specificity $=64.65 \%$, Accuracy $=55.10 \%, M R=44.90 \%$, $P P V=36.52 \%, N P V=65.61 \%, L R+=1.0608, L R-=0.9667, R R=1.0618$

\section{Table 14: Crosstabulation of observed and predicted frequencies of neonatal status}

\begin{tabular}{|l|c|r|r|r|}
\hline \multicolumn{5}{|c|}{ predicted * observed Crosstabulation } \\
\hline Count & \multicolumn{3}{|c|}{ observed } & \multirow{2}{*}{ Total } \\
\cline { 3 - 5 } \multicolumn{2}{|c|}{} & alive & \multicolumn{1}{c|}{ dead } & \\
\hline \multirow{2}{*}{ predicted } & alive & 2513 & 9 & 2522 \\
\cline { 2 - 5 } & dead & 1037 & 8 & 1045 \\
\hline Total & 3550 & 17 & 3567 \\
\hline
\end{tabular}

Cross tabulation of observed and predicted frequencies of neonatal status is showed in Table 14. From Table 14, the calculated indices for neonatal status are as follows:

Sensitivity $=70.79 \%$, Specificity $=47.06 \%$, Accuracy $=70.68 \%, M R=29.32 \%$, $P P V=99.64 \%, N P V=0.01 \%, L R+=1.3372, L R-=0.6207, R R=1.0041$

\section{DISCUSSION}

\section{Multinomial Logistic Regression Model}

MLR model consists of variables maternal age, number of previous caesarian sections, number of previous births, estimate gestational age, Pre-Eclampsia, number of previous pregnancies, Placenta Preavia and Abruption Placenta; which are useful predictors for distinguishing between categories of onset of labour. The fitted MLR model is able to accurately classify an individual according to labour category (Proportional by Chance Accuracy Rate for IOL classification $=65.5 \%$ ). According to the $\mathrm{R}^{2}$ value (Table 7 ), $37.8 \%$ variability of onset of labour is explained by the explanatory variables.

\section{Fuzzy Expert System}

The FES score is appropriate to distinguish whether an individual should go through IOL or not. It also can be used to identify whether a new born would survive after seven days of life. This score was obtained from membership functions of the factors including maternal age, total number of neonates delivered, birth weight, Maternal Severity Index (MSI), 
estimate gestational age, number of previous caesarian sections, number of previous pregnancies and number of previous births respectively.

The sensitivity and specificity of the Fuzzy Expert System for IOL (Table 13) is $37.50 \%$ and $64.65 \%$ respectively. The accuracy of that system is $55.10 \%$. For the Fuzzy Expert System for neonatal status, sensitivity is $70.79 \%$, specificity is $47.06 \%$ and the accuracy is $70.68 \%$ (Table 14). Therefore, if the parameters are known, these two systems can be used to determine whether a specific mother should go through IOL and also whether a neonate will be alive after seven days of birth.

Using the FES, onset of labour category can be identified according to following criteria: if score $<0.8570$ then onset of labour category is spontaneous; if $0.8570 \leq$ score $\leq$ 0.8881 then onset of labour category is induced, and if score $>0.8881$ then onset of labour category is no labour.

A combination of these two models, can be used to assess the association between IOL and each pregnancy outcome: if $0.8570 \leq$ score $\leq 0.8854$, onset of labour is IOL and neonatal status is alive; and if $0.8854 \leq$ score $\leq 0.8881$, onset of labour is IOL and neonatal status is dead.

\section{Limitations of the Study}

Given that the study is uses previously collected data, IOL predictive variables related to previous health conditions of pregnant women, maternal complication type, maternal Body Mass Index (BMI), and facilities available at the hospital are absent. Inclusion of these variables could improve the predictive value of the methods described.

\section{CONCLUSIONS AND RECOMMENDATIONS}

MLR and FES models were used to predict IOL outcome. The MLR model predict IOL with a classification rate of $65.5 \%$ and the FES predict IOL with an accuracy of $55.10 \%$. Maternal age, number of previous caesarian sections, number of previous births, estimated gestational age, Pre-Eclampsia, number of previous pregnancies, Placenta Preavia, Abruption Placenta, total number of neonates delivered, birth weight and MSI were identified as factors associated with IOL. Neonatal status after seven days of life can also be predicted using the developed FES. If the FES score is $0.8570 \leq$ score $\leq 0.8854$, then the

patient would be within the induced group and the baby would be alive after seven days of 
birth. We conclude that MLR and Fuzzy models can be used to drive decision making procedures related to IOL.

Both FES and MLR model can be improved by introducing new variables, such as Apgar score, Bishop score, variables related to previous health indicators of pregnant women, variables related to each maternal complication type, maternal Body Mass Index (BMI) of pregnant women, and facilities available at the hospital.

\section{REFERENCES}

Azim, T., Jaffar, M. A., \& Mirza, A. M. (2014). Fully automated real time fatigue detection of drivers through Fuzzy Expert Systems. Applied Soft Computing Journal, 18, 25-38. https://doi.org/10.1016/j.asoc.2014.01.020

Bayrampour, H., Salmon, C., Vinturache, A., \& Tough, S. (2015). Effect of depressive and anxiety symptoms during pregnancy on risk of obstetric interventions. Journal of Obstetrics and Gynaecology Research, 41(7), 1040-1048. https://doi.org/10.1111/jog.12683

Gawade, P. L., \& Whitcomb, B. W. (2012). Maternal and fetal factors associated with labor and delivery complications. http://scholarworks.umass.edu/open_access_dissertations/503/

Hiluf, S., \& Assefa, Y. (2015). Addis ababa, ethiopia 0, (June). How to perform a Multinomial Logistic Regression in SPSS Statistics | Laerd Statistics. (n.d.). https://statistics.laerd.com/spsstutorials/multinomial-logistic-regression-using-spssstatistics.php

Laws, P. J., Li, Z., \& Sullivan, E. A. (2010). Australia's mothers and babies 2008. Australian Institute of Health and Welfare, 130.

Nascimento, L. F. C., \& Ortega, N. R. S. (2002). Fuzzy linguistic model for evaluating the risk of neonatal death. Revista de Saude Publica, 36(6), 686-692. https://doi.org/10.1590/S0034-89102002000700005

National Institute for Health and Clinical Excellence: Guidance. (2008). In Induction of Labour. RCOG Press. Retrieved from http://www.ncbi.nlm.nih.gov/pubmed/21510026 Pappis, C. P. (2005). Fuzzy Reasoning. Search Methodologies: Introductory Tutorials in Optimization and Decision Support Techniques, 38. https://doi.org/10.1007/0-38728356-0_15 
Reis, M. A. M., Ortega, N. R. S., \& Silveira, P. S. P. (2004). Fuzzy expert system in the prediction of neonatal resuscitation. Brazilian Journal of Medical and Biological Research, 37(5), 755-764. https://doi.org/10.1590/S0100-879X2004000500018

WHO UNICEF UNFPA World Bank Group and United and United Nations Population Division. (2015). Philippines: Maternal mortality in 1990-2015, 1-5. https://doi.org/http://www.who.int/gho/maternal_health/countries/sle.pdf

World Health Organization. (2011). WHO recommendations for Induction of labour. World Health Organization, 1-39. https://doi.org/10.1016/0140-6736(93)92727-B 